\title{
Morphologische Untersuchung der Schweißdrüsen bei Menschen, insbesondere über das Vorkommen ihrer abnormen Form.
}

$$
\text { Von }
$$

\section{Fujio Takayama}

Aus dem Anatomischen Institut der Keio Universität, Tokyo.

Die Morphologie der ekkrinen und apokrinen Schweißdrüsen bei den Menschen, besonders das Vorkommen der Divertikel-, Schlingenund Anastomosenbildung, ein Drüsenkörper mit 2 Ausführungsgängen und ein gemeinsamer Ausführungsgang mit 2 Drüsenkörpern ist von Clausen und Alexanderson (1929), Alverdes (1932), Sperling (1935), Peter (1935), Horn (1935), Morioka (1936) und Aurell (1938) untersucht und umstritten. Aber bezüglich des Auftretens dieser abnormen Formen stimmen die Meinungen $z$ wischen den Autoren nicht überein.

Ich habe bei der quantitativen Untersuchung der Schweißdrüsen von 600 Drüsenkörpern und ihren Ausführungsgängen die Gelegenheit gehabt, die Verhältnisse zwischen dem Drüsenkörper und Ausführungsgang $z \mathrm{u}$ beobachten.

Das gebrauchte Material sind Flächenschnittserienpräparate von Hautstücken in ca. $1.2 \mathrm{qcm}$ Größe. Ihre Schnittdicke ist $40 \mu$. Die Färbung geschah mit Hämatoxylin (Hansen)-Eosin-Doppelfärbung. Für die Erklärung wurden nur Wachsmodelle hergestellt. (Fig. 1-15).

\section{Eigene Befunde.}

Die Beziehung zwischen dem Drüsenkäuel der Schweißdrüsen und der Form des Ausführungsganges sind sehr mannigfaltig.

Ich habe nach der Form des Drüsenknäuels, der Verzweigung des Ausführungsganges und der Verwachsung des Knäuels die folgenden 
Typen unterschieden.

1. Einteilung nach der Form des Knäuels.

Typus A. Einfaches Knäuel bildender Drüsenkörper (normale Form, Fig. 1, 2 und 4).

Typus B. Vom Drüsenkörper ausgehender langer Ausläufer des Drüsenkanälchen, welches mit einem blinden Ende endet (Fig. 6).

Typus C. Vom Drüsenkörper ausgehendes Drüsenkanälchen, welches wieder ein Knäuel bildet (Fig. 7).

2. Einteilung nach der Verzweigungsweise des Ausführungsganges.

Typus D. Der von einem Drüsenkörper ausgehende eine Ausführungsgang teilt sich in seinem Verlaufe in 2 Gänge, und die beiden Gänge münden mit selbstständiger Ausmündung in der Hautoberfläche (Fig. 11, 16).

Typus E. Ein Ausführungsgang teilt sich in seinem Verlaufe in 2 Gänge, die einen selbstständigen Drüsenkörper besitzen (Fig. 10).

3. Einteilung nach der Verwachsung der Knäuel.

Typus F. 2 oder über 2 Drüsenkörper vereinigen sich miteinander und bilden scheinbar einen großen Drüsenkörper (Fig. 12, 13, 14 und 17).

Typus G. Voneinander isolierende 2 oder über 3 Drüsenkörper sind mit:einem Drüsenkanälchen verbunden (Fig. 15, 19, 20, 21, 22 und 23).

Typus A ist die gewöhnliche Form des Drüsenkörpers der Schweißdrüse. Die Drüse besitzt nur einen Ausführungsgang. Wie in der Figur 1 ersichtlich ist, nimmt im allgemeinen der Ausführungsgang im. Drüsenkörper den oberen Teil des. Knäuels ein und das Sekretionskánälchen den unteren Abschnitt desselben. Bei diesem Fall umschlingen sich der Ausführungsgang und die Sekretionskanälchen miteinander und bilden ein Drüsenknäuel.

Der Ausführungsgang geht im allgemeinen von dem oberen Teil des Drüsenkörpers, d.h., dem der Hautoberfläche nahen Teil aus; mitunter nimmt er seinen Ausgang von den verschiedenen Abschnitten des Drüsenkörpers. Die Figur 4 zeigt den Ausgang des Ausführungsganges vom untersten Teil des Drüsenkörpers. Mitunter durchzieht der Ausführungsgang in seinem Verlaufe den Drüsenkörper anderer Drüsen und erreicht den eigenen Drüsenkörper (Fig. 5).

Fig. 3 zeigt, daß ein Ausführungsgang von ein Sekretionskanälchen bildenden Knäuel ausgeht, dann wieder ein kleines Knäuel bildet. Man findet auch das Bild vor, wo das Sekretionskanälchen von dem Drüsenknäuel ausgeht, die Nähe des Blutgefäßes erreicht und dasselbe umschlingt (Fig. 8 und 18). Auch zeigt sich der Befund, nach welchem 
das Sekretionskanälchen das Blutgefä $B$ als Achse, in seiner Umgebung eine Schlinge bildet. Von diesen Befunden kann man verstehen, daß zwischen den Drüsen und den Blutgefäßen innige Beziehungen bestehen.

Tabelle 1 stellt die abnorme Beziehung zwischen dem Drüsenkörper und dem Ausführungsgang und die Häufigkeit des Auftretens jedes Typus dar. Die in der Tabelle angegebene Zahl ist die in 100 Drüsenindividuen gefundene $Z a h l$; dementsprechend kann man diesen Wert zugleich mit Prozentsatz annehmen. Diese abnorme Form wird am Kopf reichlich aufgefunden. Besonders beim Typus $F$ ist ihre Neigung $\mathrm{zu}$ sehen. Am Bauch ist eine solche Form nur einmal aufgefunden, aber niemals an der Beugeseite des Oberarms.

Aus den obigen Befunden kann man schließen, daß diese abnorme Form wahrscheinlich in den die Drüsenzahl in einem bestimmten Hautflächeninhalt reichlich vorkommenden Körperteile, wie Kopf, häufig vorkommt.

Unter 10 Körperteilen ist diese abnorme Form am Kopf (20) am häufigsten, dann Vorderarm (8), Unterschenkel (8), Brust (5), Oberschenkel (5), Unterschenkel (4), Rücken (4), Hals (2) und Bauch (1); am Oberarm. ist sie garnicht auffindbar (Tabelle 1).

Die Menge der scheinbar einen Drüsenkörper, aber zwei Ausführungsgänge besitzenden Drüse zeigt die Tabelle 2.

Aus der Tabelle 2 ist klar ersichtlich, daß das Volumen der Drüse mit 2 Ausführungsgängen in 8 unter 40 Drüsen über das 2 fache des durchschnittlichen Volumens der einen Drüse beträgt. In 26 Drüsen ist es unter dem 2 fachen durchschnittlichen Volumen der einen Drüse. In 6 Drüsen ist es kleiner als das durchschnittliche Volumen einer Drüse. Es betrifft diese Befunde häufig am Kopf. Die Häufigkeit einer solchen abnormen Drüse (d. h. Drüse mit 2 Ausführungsgängen) am Kopf steht mit dem Alter in keinem Zusammenhang.

\section{Zusammenstellung.}

Typus A, bei welchem jede Drüse nur einen eigenen Ausführungsgang aufweist, ist die gewöhnliche Drüsenform. Wie Figur 6 zeigt, habe ich einen Fall (Typus B) gefunden, welcher aus dem Drüsenknäuel ein blind endendes Kanälchen abgehen läßt.

Über das Vorkommen der Typus $D$, bei welchem ein und dieselbe Drüse zwei (geteilte) Ausführungsgänge besitzt, gehen die Angaben der verschiedenen Autoren vollständig auseinander. Clausen und Alexanderson berichteten im Jahre 1929 und 1932, daß ein und dieselbe Drüse zwei oder mehr Ausführungsgänge besitzt, sie fanden eine solche Drüse in ein Drittel bis ein Viertel aller untersuchten Fälle. Wagner (1939) hat ebenfalls die Drüse mit zwei deutlichen Ausführungsgängen. 
Während ganz entgegengesetzt der Meinung von Clausn und Alexanderson, Horn ('35), Aurell ('38) berichteten, daß ein und dieselbe Drüse immer einen eigenen Ausführungsgang besitzt.

Den Typus $\mathrm{E}$, bei welchem zwei oder mehr Drüsen einen gemeinsamen Ausführungsgang besitzen, habe ich in 6 von 1000 Drüsenindividuen gefunden. Über das Vorhandensein dieses Typus schreibt Kra use (1844): "Zuweilen vereinigten sich die Tubuli zweier Drüsen zu einem gemeinschaftlichen Ausführungsgang". Er ist aber der Meinung, da $\beta$ dieses Verhalten recht selten sei. Kölliker (1889) äußert sich in gleichen Sinne. Nach der Untersuchung von Aurell (1938) anastomisiert die Drüse mit anderen Drüsen und hat nie einen gemeinsamen Ausführungsgang mit diesen. Diesbezüglich möchte ich sagen, daß auf dem Wege der Entwicklung abnormalerweise die Anlage des einen Ausführungsganges in zwei oder mehr geteilt werden könne. Diese Ansicht ist heute von vielen Embryologen vertreten.

Auch über die Verzweigung, Anastomose- und Divertikelbildung der Drüse gehen die Meinungen der Autoren auseinander. Diese Frage wird von Krause (1884), Pinkus ('27), Horn ('35) und Aurell ('38) verneint, dagegen von Clausen und Alexanderson (1932) und Morioka (1936) angenommen.

Bezüglich der apokrinen Drüse fanden Sperling ('35), Alverdes ('32) und Morioka die Verästelung, Anastomose (Ringbildung) und Ausbuchtungen (Divertikel) der Drüse, dagegen kam Peter ('35) zu einem vollständig entgegengestztem Resultat.

Aus meinen Befunden läßt sich nicht mit Sicherheit sagen, da $B$ die wirkliche Verästelung, Anastomose zweier Drüsen, Vereinigung der beiden Ausführungsgänge sicher vorhanden oder nicht vorhanden ist.

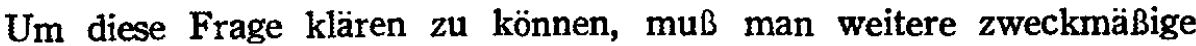
Untersuchungen mit Hilfe von verschiedenen Methoden unternehmen.

\section{Benüitzte Literaturen.}

1) Wagner, R.: Icones physiologicae. Wien, 1839.

2) Krause: Wagners' Handwörterbuch der Physiologie. Bd. 2 (Haut), S. 127. Braunschweig, 1844 .

3) Krause: Wagner's Handb. d. Physiol. Bd. 2 (Haut), S. 127, 1874.

4) Kölliker, A.: Handbuch der Gewebelehre des Menschen. 6. Auf. 1889.

5) Pinkus, F.: Normale Anatomie der Haut. Handbuch der Haut-und Ceschlechtskrankheiten. Teil $1 / 1$, S. 300, Berlin, 1927.

6) Clausen und Alexanderson: Z. mikrosk.-anat. Forsch. Bd. 18, S. 47, 1929; Bd. 30 , S. $175,1932$.

7) Alverdes, K.: Z. mikrosk.-anat. Forsch. Bd. 28, S. 609, 1932. 
8) Sperling, G.: Z. mikrosk.-anat. Forsch. Bd. 38, S. 241, 1935.

9) Peter, K. und Horn, G.: Z. mikrosk.-anat. Forsch Bd. 38, S. 471, 1935.

10) Horn, G.: Z. mikrosk--anat. Forsch. Bd. 38, S. 318, 1935.

11) Morioka, Y.: Okayama-Igakkai-Zasshi Jg. 48. Nr. 1. J. 1936 (Japanisch).

12) Aurell, G.: Z. mikrosk.-anat. Forsch. Bd. 44. S. 56, 1938. 
Tafel I
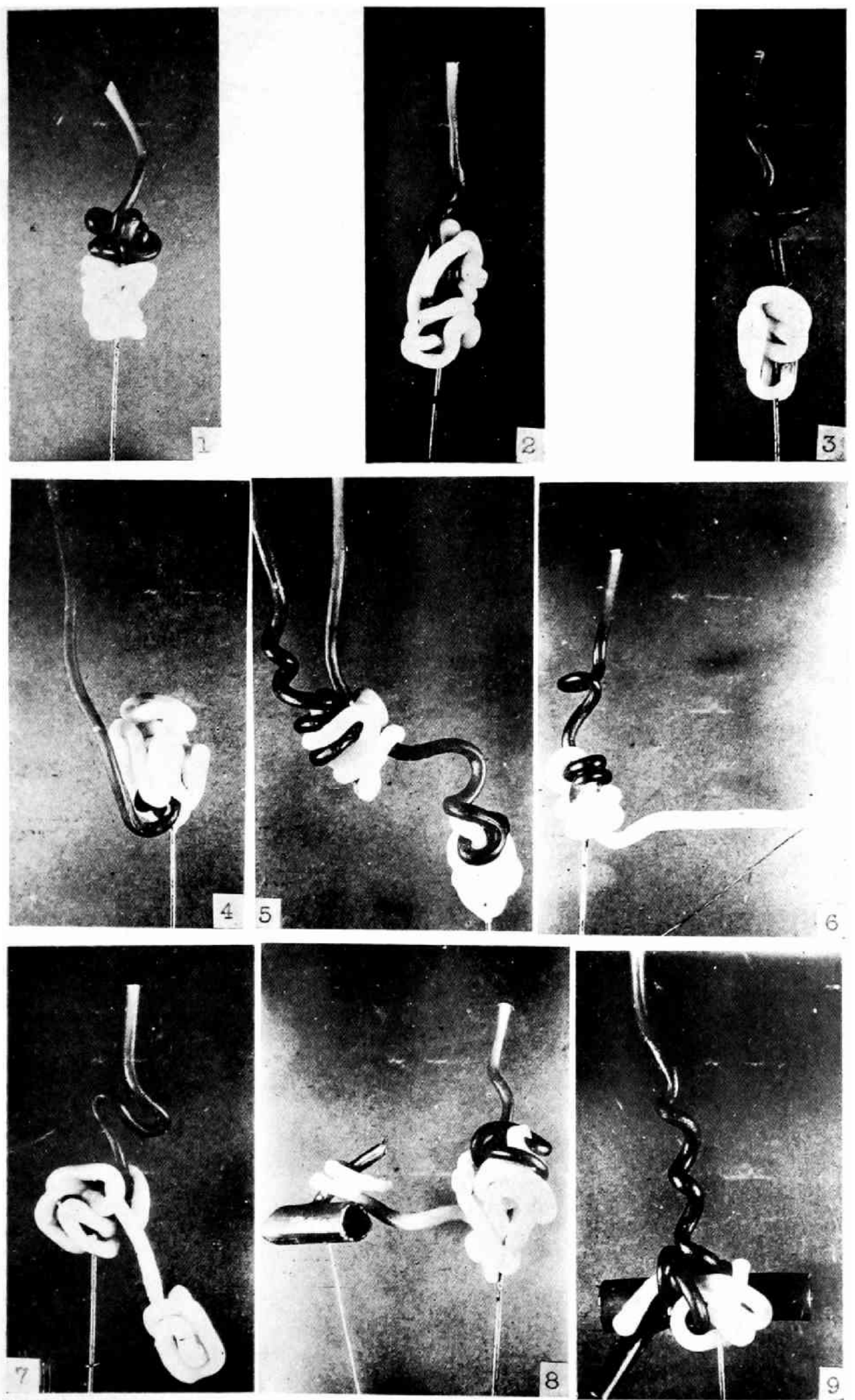

F. Takayama

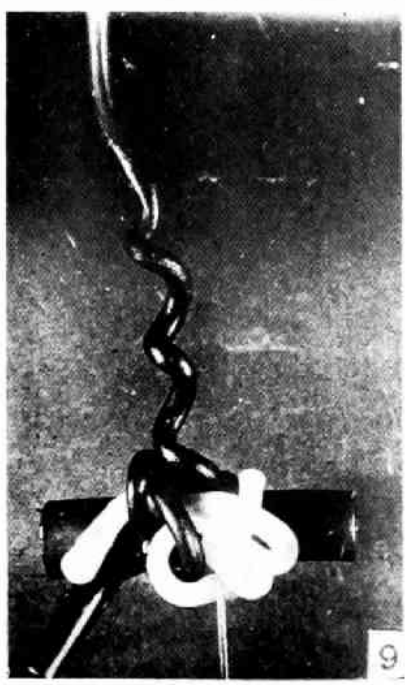


Tafel II
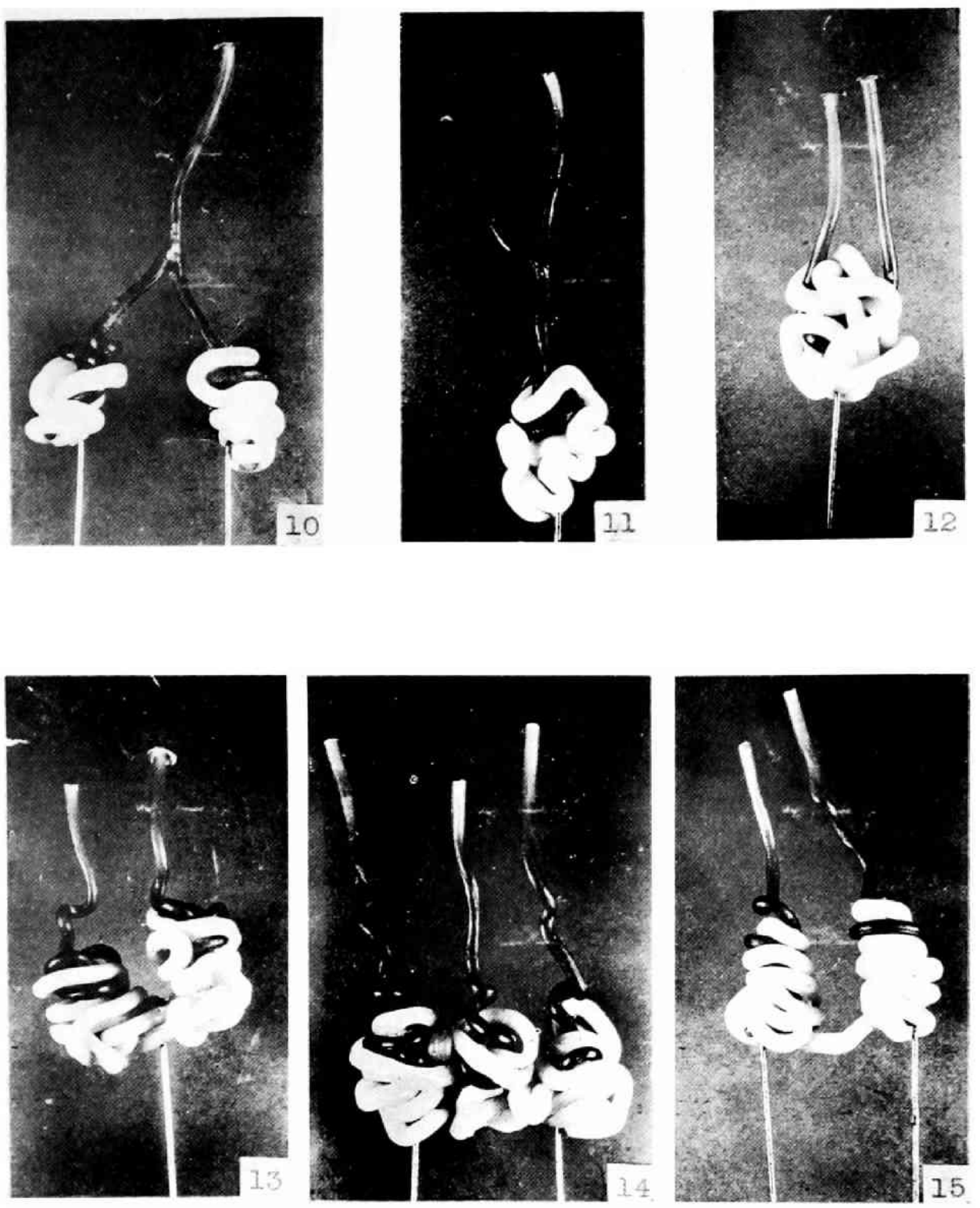

F. Takayama 



F. Takayama 

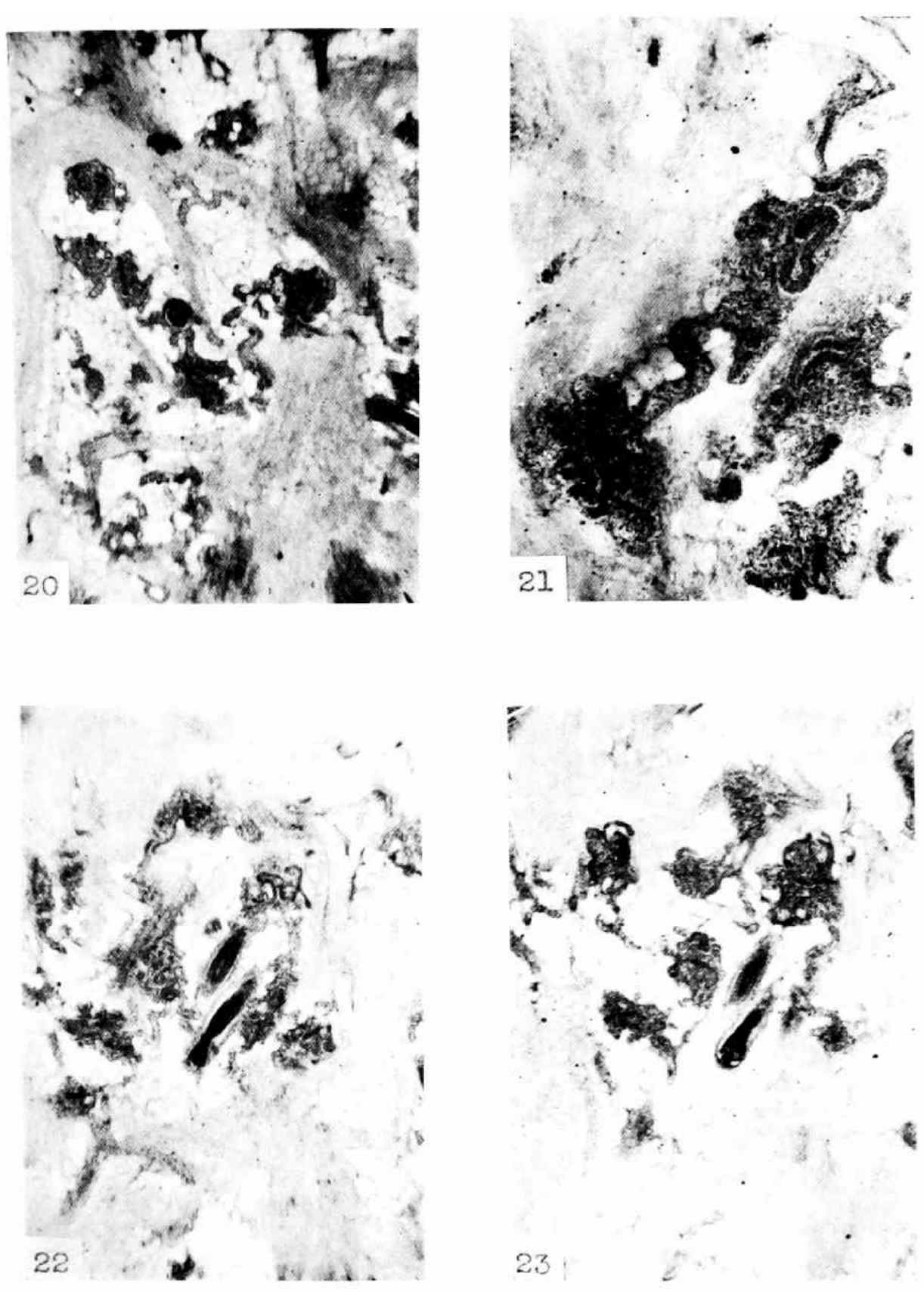

F. Takayama 